\title{
Minimum-Latency Beaconing Schedule in Multihop Wireless Networks
}

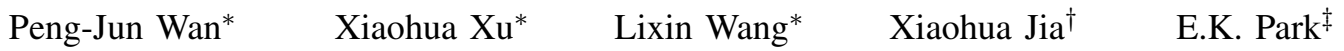

\begin{abstract}
Minimum-latency beaconing schedule (MLBS) in synchronous multihop wireless networks seeks a schedule for beaconing with the shortest latency. This problem is NP-hard even when the interference radius is equal to the transmission radius. All prior works assume that the interference radius is equal to the transmission radius, and the best-known approximation ratio for MLBS under this special interference model is 7 . In this paper, we present a new approximation algorithm called strip coloring for MLBS under the general protocol interference model. Its approximation ratio is at most 5 when the interference radius is equal to transmission radius, and is between 3 and 6 in general.
\end{abstract}

\section{INTRODUCTION}

Beaconing in wireless networks is a primitive communication task in which every node locally broadcasts a packet to all its neighbors. Assume that all communications proceed in synchronous time-slots and each node can transmit at most one packet of a fixed size in each time-slot. A beaconing schedule assigns a time-slot to every node subject to the constraint that the nodes assigned in each time-slot are interference free. The latency of a beaconing schedule is the number of time-slots during which at least one transmission occurs. The problem of computing a beaconing schedule with minimum latency in a wireless network is referred to Minimum-Latency Beaconing Schedule (MLBS). The problem MLBS is a classic and fundamental problem in wireless networks, and variants of this problem have been extensively studied in [1], [2], [4], [10], [11], [13], [14], [15], [16].

In this paper, we study the problem MLBS under the following model for wireless networks. All the networking nodes are located in a plane and are each equipped with an omnidirectional antenna. Each node has a fixed transmission radius which is normalized to one and an interference radius $\rho \geq 1$. The communication range and the interference range of a node $v$ are the two disks centered at $v$ of radius one and $\rho$ respectively (see Figure 1). A node $v$ can receive the message successfully from a transmitting node $u$ if $v$ is within

*Department of Computer Science, Illinois Institute of Technology. Emails: wan@cs.iit.edu, xxu23@iit.edu, and wanglix@iit.edu. This work was supported in part by National Science Foundation of USA under grant CNS0831831.

$\dagger$ Department of Computer Science, City University of Hong Kong. Email: jia@cs.cityu.edu.hk. This work was supported in part by Research Grants Council of Hong Kong Grant No. CityU 114307 and NSF China Grant No. 60633020.

$\ddagger$ School of Computing and Engineering, University of Missouri at Kansas City. Email: ekpark@umkc.edu. the transmission range of $u$ but is outside the interference range of any other transmitting node. Such interference model is referred to as the protocol interference model [7] and is widely used because of its generality and tractability.

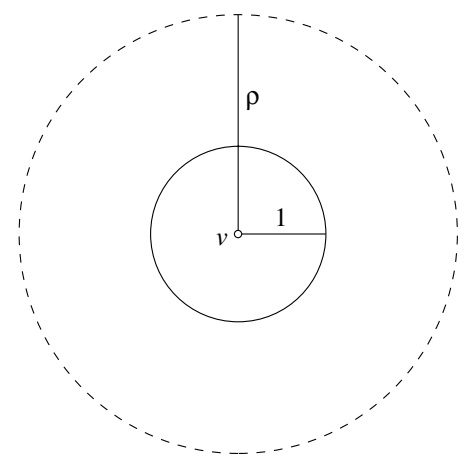

Fig. 1. The protocol interference mode: each node has a unit transmission radius and an interference radius $\rho \geq 1$.

Under the above networking model, the communication topology of a wireless network in a unit-disk graph [3] in which there is an edge between two nodes if and only if their distances is at most one. We assume that the communication topology is always connected. Then, any pair of nodes with distance at most $\max \{1, \rho-1\}$ interfere with each other, and pair of nodes with distance greater than $\rho+1$ do not interfere with each other. We also define the interference topology of a wireless network as follows: For any pair of nodes $u$ and $v$, there is an edge between $u$ and $v$ in the interference topology if one of the three conditions holds: (1) $u$ and $v$ are within each other's communication range (see Figure 2(a)), (2) some node $w$ other than $u$ and $v$ is within $u$ 's communication range and $v$ 's interference range (see Figure 2(b)), and (3) some node $w$ other than $u$ and $v$ is within $v$ 's communication range and $u$ 's interference range (see Figure 2(c)). Then a beaconing schedule for a wireless network is equivalent to a proper vertex coloring of its interference topology with the latency corresponding to the number of colors. Hence, the problem MLBS for a wireless network seeks the minimum vertex coloring of its interference topology.

All the prior works on MLBS [1], [2], [4], [10], [11], [13], [14], [15], [16] implicitly assumed that $\rho=1$. When $\rho=1$, the interference topology is the square of a unit-disk graph representing the communication topology. Sen and Huson [13] proved that minimum coloring of a square of a unit-disk graph 


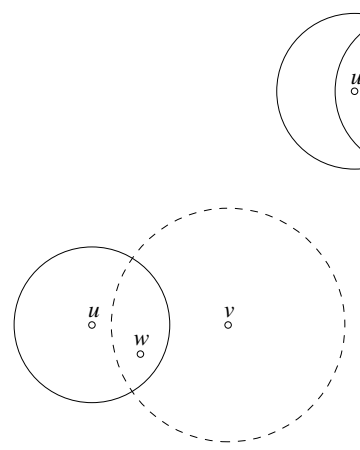

(b)

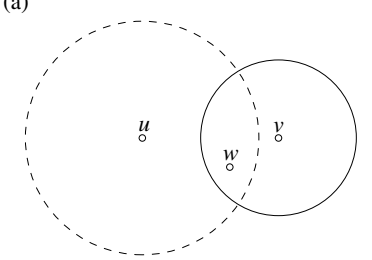

(c)
Fig. 2. Three possible scenarios of intereference between a pair of nodes $u$ and $v$.

is NP-hard. The classical greedy coloring known as first-fit coloring in the smallest-degree-last ordering [8] was adopted by Sen and Malesinska [14] to produce a coloring of the square of unit-disk graph. While this greedy coloring has polynomial approximation ratio when applied to general graphs, it is a 7approximation when restricted to squares of unit-disk graphs [16]. The bound 7 is the best-known upper bound on the achievable approximation ratio for MLBS with $\rho=1$. It has been open for years whether there is an algorithm for MLBS with $\rho=1$ whose approximation ratio is smaller than 7 .

In this paper, we propose an algorithm called strip coloring for MLBS with arbitrary $\rho \geq 1$, which exploits a key structural property of the interference topology. For $\rho=1$, its approximation ratio is at most 5 , which is smaller than the best-known bound 7 on achievable approximation ratio. For $\rho>1$, its approximation ratio ranges from 3 to 6 depending on the value of $\rho$. The following notations will be used throughout this paper. The Euclidean distance between two nodes $u$ and $v$ is denoted by $\|u v\|$. The disk of radius $r$ centered at a node $v$ is denoted by $D(v, r)$. The topological boundary of a point set $A$ is denoted by $\partial A$. Let $G=(V, E)$ be an arbitrary graph. We use $\chi(G)$ and $\omega(G)$ to denote its chromatic number and clique number of $G$ respectively. In general, $\chi(G) \geq \omega(G)$. If $\chi(G)=\omega(G)$, then $G$ is said to be perfect.

The remaining of this paper is organized as follows. In Section II, we introduce a function and present several properties of this function. In Section III, we describe the algorithm strip coloring and analyze its approximation ratio. Finally, we conclude this paper in Section III by discussing on some further relevant results.

\section{PREliminaries}

Define a function $h(\rho)$ over $[1, \infty)$ as follows: if $1 \leq \rho<$ $\sqrt{\frac{3+\sqrt{13}}{2}}$, then

$$
h(\rho)=\cos \frac{\arccos \frac{\rho}{2}+\arccos \frac{1}{2 \rho}}{2}
$$

$$
\text { if } \begin{aligned}
\sqrt{\frac{3+\sqrt{13}}{2}} & \leq \rho \leq 2 \text {, then } \\
& h(\rho)=\sin \left(\arccos \frac{1}{2 \rho}-\arcsin \frac{1}{\rho}\right) ;
\end{aligned}
$$

if $\rho>2$, then

$$
h(\rho)=(\rho-1) \sin \left(\arccos \frac{\rho-1}{2 \rho}-\arcsin \frac{1}{\rho}\right) .
$$

A straightforward calculation yields that if $1 \leq \rho<\sqrt{\frac{3+\sqrt{13}}{2}}$, then

$$
h(\rho)=\sqrt{\frac{5}{8}-\frac{1}{4} \sqrt{\left(\frac{5}{2}\right)^{2}-\left(\rho+\frac{1}{\rho}\right)^{2}}} ;
$$

if $\sqrt{\frac{3+\sqrt{13}}{2}} \leq \rho \leq 2$, then

$$
h(\rho)=\sqrt{\left(1-\frac{1}{4 \rho^{2}}\right)\left(1-\frac{1}{\rho^{2}}\right)}-\frac{1}{2 \rho^{2}} ;
$$

if $\rho>2$, then

$$
h(\rho)=\frac{(\rho-1)^{2}}{2 \rho^{2}}\left((\rho+1) \sqrt{\frac{3 \rho-1}{\rho-1}}-1\right) .
$$

We first present the geometric interpretation of the value $h(\rho)$.

Lemma 1: Suppose that $1 \leq \rho \leq \sqrt{\frac{3+\sqrt{13}}{2}}$. Let $p q$ be a line segment with $\rho-1<\|p q\|<2$. $u$ and $v$ are the two points on the opposite side of $p q$ such that $\|u p\|=\|u q\|=\|v q\|=1$ and $\|v p\|=\rho$ (see Figure 3). Then the distance between $q$ and $u v$ strictly increases with $\|p q\|$ and equals to $h(\rho)$ when $\|p q\|=\rho$.

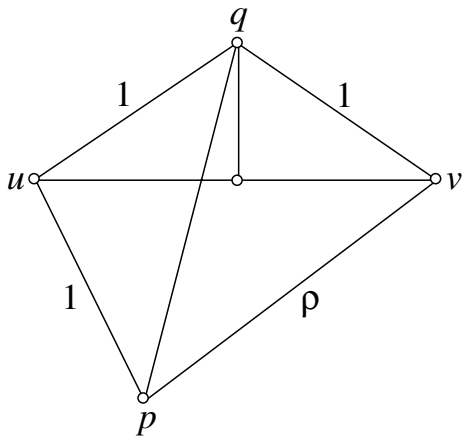

Fig. 3. Figure for Lemma 1.

Proof: By law of cosine,

$$
\cos \angle p q v=\frac{\|p q\|^{2}+1-\rho^{2}}{2\|p q\|}=\frac{1}{2}\left(\|p q\|-\frac{\rho^{2}-1}{\|p q\|}\right) .
$$

Since $\rho \geq 1, \cos \angle p q v$ strictly increases with $\|p q\|$ when $0<\|p q\|<2$. So, $\angle p q v$ strictly decreases with $\|p q\|$. Clearly, $\angle p q u$ strictly decreases with $\|p q\|$. So, $\angle u q v$ strictly decreases with $\|p q\|$. Thus, the distance between $q$ and $u v$ strictly increases with $\|p q\|$. 
When $\|p q\|=\rho$, we have

$$
\angle u q v=\angle p q u+\angle p q v=\arccos \frac{\rho}{2}+\arccos \frac{1}{2 \rho} .
$$

Hence the distance between $q$ and $u v$ is

$$
\cos \frac{\angle u q v}{2}=h(\rho) .
$$

Lemma 2: Suppose that $\rho \geq \sqrt{\frac{3+\sqrt{13}}{2}}$. Consider a right triangle $\triangle p u v$ with $\angle p u v=\frac{\pi}{2},\|p u\|=1$ and $\|p v\|=\rho$ (see Figure 4). Let $q$ be a point on the circle $\partial D(v, \max \{1, \rho-1\})$ which lies on the different side of $u v$ from $p$ and $\angle u v q \leq \frac{\pi}{2}$. Then the distance between $q$ and $u v$ strictly decreases with $\|p q\|$, and equals to $h(\rho)$ when $\|p q\|=\rho$.

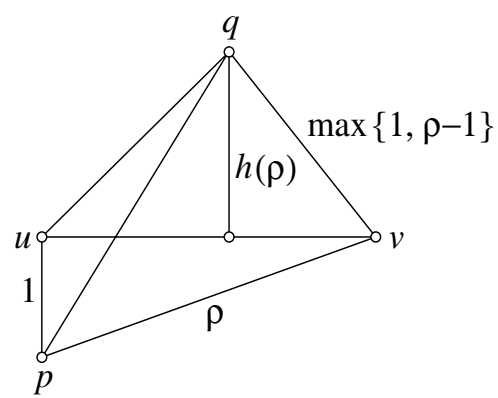

Fig. 4. Figure for Lemma 2.

Proof: By law of cosine, $\angle p v q$ strictly increases with $\|p q\|$. Since $\angle u v q=\angle p v q-\angle p v u$ and $\angle p v u$ is fixed, $\angle u v q$ also strictly increases with $\|p q\|$, and so does the distance between $q$ and $u v$. When $\|p q\|=\rho$,

$$
\begin{aligned}
\angle u v q & =\angle p v q-\angle p v u \\
& =\arccos \frac{\max \{1, \rho-1\}}{2 \rho}-\arcsin \frac{1}{\rho},
\end{aligned}
$$

and hence the distance between $q$ and $u v$ is

$$
\max \{1, \rho-1\} \sin \angle u q v=h(\rho) .
$$

Next, we give some useful properties of $h(\rho)$. We begin with the following lemma.

Lemma 3: $\arccos \frac{\rho-1}{2 \rho}-\arcsin \frac{1}{\rho}$ strictly increases with $\rho$ when $\rho \geq 1$.

Proof: Let

$$
\theta=\arccos \frac{\rho-1}{2 \rho}-\arcsin \frac{1}{\rho} .
$$

Set $t=\frac{1}{\rho}$. Then, $0<t \leq 1$ and

$$
\theta=\arccos \frac{1-t}{2}-\arcsin t
$$

and the derivative of $\theta$ over $t$ is

$$
\begin{aligned}
\frac{d \theta}{d t} & =-\frac{-\frac{1}{2}}{\sqrt{1-\left(\frac{1-t}{2}\right)^{2}}}-\frac{1}{\sqrt{1-t^{2}}} \\
& =\frac{1}{2 \sqrt{1-\left(\frac{1-t}{2}\right)^{2}}}-\frac{1}{\sqrt{1-t^{2}}} \\
& =\frac{1}{\sqrt{(3-t)(1+t)}}-\frac{1}{\sqrt{(1-t)(1+t)}} \\
& =\frac{1}{\sqrt{1+t}}\left(\frac{1}{\sqrt{3-t}}-\frac{1}{\sqrt{1-t}}\right) . \\
& <0 .
\end{aligned}
$$

Hence, $\frac{d \theta}{d t}<0$ when $0<t<1$. This implies that $\theta$ strictly decreases with $t$ when $0<t \leq 1$,and consequently strictly increases with $\rho$ when increases $\rho \geq 1$.

It's easy to verify that $h(\rho)$ is continuous. The lemma below shows the monotonicity of $h(\rho)$.

Lemma 4: $h(\rho)$ is strictly increasing. In addition, $\frac{h(\rho)}{\rho+1}$ is also strictly increasing when $\rho \geq 2$.

Proof: When $1 \leq \rho \leq \sqrt{\frac{3+\sqrt{13}}{2}}$,

$$
h(\rho)=\sqrt{\frac{5}{8}-\frac{1}{4} \sqrt{\left(\frac{5}{2}\right)^{2}-\left(\rho+\frac{1}{\rho}\right)^{2}} .}
$$

Since $\rho+\frac{1}{\rho}$ strictly increases when $\rho \geq 1, h(\rho)$ strictly increases when $1 \leq \rho \leq \sqrt{\frac{3+\sqrt{13}}{2}}$.

When $\sqrt{\frac{3+\sqrt{13}}{2}} \leq \rho \leq 2$,

$$
h(\rho)=\sin \left(\arccos \frac{1}{2 \rho}-\arcsin \frac{1}{\rho}\right),
$$

Hence, $h(\rho)$ strictly increases when $\sqrt{\frac{3+\sqrt{13}}{2}} \leq \rho \leq 2$.

When $\rho>2, h(\rho)$ strictly increases. Since $\frac{\rho-1}{\rho+1}$ strictly increases and

$$
\frac{h(\rho)}{\rho+1}=\frac{\rho-1}{\rho+1} \sin \left(\arccos \frac{\rho-1}{2 \rho}-\arcsin \frac{1}{\rho}\right),
$$

$\frac{h(\rho)}{\rho+1}$ strictly increases with $\rho$ when $\rho>2$ by Lemma 3 .

Next, we give some inequalities satisfied by $h(\rho)$.

Lemma 5: The following statements are true.

1) For any $\rho \geq 1$,

$$
h(\rho)<\frac{\sqrt{3}}{2} \max \{1, \rho-1\} .
$$

2) For any $1<\rho \leq 2$,

$$
\frac{\rho+1}{5}<h(\rho)<\frac{\rho+1}{4} .
$$

3) When $\rho$ is less than (resp., equal to, greater than) $\sqrt{\frac{3+\sqrt{13}}{2}}, 2 \sqrt{(\max \{1, \rho-1\})^{2}-h(\rho)^{2}}$ is greater than (resp., equal to, less than) $\sqrt{\rho^{2}-1}$. 
Proof: (1) By Lemma 3, $\arccos \frac{\rho-1}{2 \rho}-\arcsin \frac{1}{\rho}$ strictly increases on $[2, \infty)$. Since

$$
\lim _{\rho \rightarrow \infty}\left(\arccos \frac{\rho-1}{2 \rho}-\arcsin \frac{1}{\rho}\right)=\frac{\pi}{3} .
$$

we have

$$
\arccos \frac{\rho-1}{2 \rho}-\arcsin \frac{1}{\rho}<\frac{\pi}{3}
$$

for any $\rho \geq 2$. Thus when $\rho \geq 2$,

$$
\begin{aligned}
h(\rho) & =(\rho-1) \sin \left(\arccos \frac{\rho-1}{2 \rho}-\arcsin \frac{1}{\rho}\right) \\
& <(\rho-1) \sin \frac{\pi}{3}=\frac{\sqrt{3}}{2}(\rho-1) .
\end{aligned}
$$

Since $h(\rho)$ strictly increases on $[1,2]$ by Lemma 4 , we have

$$
h(\rho) \leq h(2)<\frac{\sqrt{3}}{2} .
$$

for any $1 \leq \rho \leq 2$. So the first part of the lemma holds.

(2) We prove the second part in two cases.

Case 1: $1 \leq \rho \leq \sqrt{\frac{3+\sqrt{13}}{2}}$. In this case,

$$
h(\rho)=\sqrt{\frac{5}{8}-\frac{1}{4} \sqrt{\left(\frac{5}{2}\right)^{2}-\left(\rho+\frac{1}{\rho}\right)^{2}}}
$$

Let $t=\rho-1$. Then, $t \in\left[0, \sqrt{\frac{3+\sqrt{13}}{2}}-1\right]$.

We first show that

$$
h(\rho)>\frac{\rho+1}{5} .
$$

Replacing $\rho$ with $t+1$, we have

$$
\begin{aligned}
& \left(\frac{5}{2}-\frac{4}{25}(\rho+1)^{2}\right)^{2}-\left(\frac{5}{2}\right)^{2}+\left(\rho+\frac{1}{\rho}\right)^{2} \\
& =\left(\frac{5}{2}-\frac{4}{25}(t+2)^{2}\right)^{2}-\left(\frac{5}{2}\right)^{2}+\left(t+1+\frac{1}{t+1}\right)^{2} \\
& =\frac{16 t^{6}+160 t^{5}+781 t^{4}+908 t^{3}+164 t^{2}+24 t+756}{625(t+1)^{2}}
\end{aligned}
$$$$
>0 \text {. }
$$

Hence,

$$
\frac{5}{2}-\frac{4}{25}(\rho+1)^{2}>\sqrt{\left(\frac{5}{2}\right)^{2}-\left(\rho+\frac{1}{\rho}\right)^{2}},
$$

which implies that

$$
\frac{5}{8}-\frac{1}{4} \sqrt{\left(\frac{5}{2}\right)^{2}-\left(\rho+\frac{1}{\rho}\right)^{2}}>\left(\frac{\rho+1}{5}\right)^{2} .
$$

So,

$$
h(\rho)>\frac{\rho+1}{5} .
$$

Now, we show that

$$
h(\rho)<\frac{\rho+1}{4} .
$$

Replacing $\rho$ with $t+1$, we have

$$
\begin{aligned}
& \left(\frac{5}{2}\right)^{2}-\left(\rho+\frac{1}{\rho}\right)^{2}-\left(\frac{5}{2}-\left(\frac{\rho+1}{2}\right)^{2}\right)^{2} \\
& =\left(\frac{5}{2}\right)^{2}-\left(t+1+\frac{1}{t+1}\right)^{2}-\left(\frac{5}{2}-\left(\frac{t+2}{2}\right)^{2}\right)^{2} \\
& =-\frac{t}{16(t+1)^{2}}\left(t^{5}+10 t^{4}+37 t^{3}+32 t^{2}-28 t-48\right) .
\end{aligned}
$$

We claim that $t^{5}+10 t^{4}+37 t^{3}+32 t^{2}-28 t-48$ is negative on $[0,0.9]$, which contains $\left[0, \sqrt{\frac{3+\sqrt{13}}{2}}-1\right]$. When $t \geq 0$, $t^{5}+10 t^{4}+37 t^{3}+32 t^{2}$ is strictly convex, and so is $t^{5}+10 t^{4}+$ $37 t^{3}+32 t^{2}-28 t-48$. Thus, the maximum of $t^{5}+10 t^{4}+$ $37 t^{3}+32 t^{2}-28 t-48$ on $[0,0.9]$ is achieved at the endpoints, which are both negative by straightforward calculation. Thus, our claim holds. Hence,

$$
\sqrt{\left(\frac{5}{2}\right)^{2}-\left(\rho+\frac{1}{\rho}\right)^{2}}>\frac{5}{2}-\left(\frac{\rho+1}{2}\right)^{2},
$$

which implies that

$$
\frac{5}{8}-\frac{1}{4} \sqrt{\left(\frac{5}{2}\right)^{2}-\left(\rho+\frac{1}{\rho}\right)^{2}}<\left(\frac{\rho+1}{4}\right)^{2} .
$$

So,

$$
h(\rho)<\frac{\rho+1}{4} .
$$

Case 2: $\sqrt{\frac{3+\sqrt{13}}{2}} \leq \rho \leq 2$. In this case,

$$
h(\rho)=\sqrt{\left(1-\frac{1}{4 \rho^{2}}\right)\left(1-\frac{1}{\rho^{2}}\right)}-\frac{1}{2 \rho^{2}} .
$$

Thus,

$$
h(\rho) \geq h\left(\sqrt{\frac{3+\sqrt{13}}{2}}\right)=\frac{\sqrt{13}-1}{4}>\frac{3}{5} \geq \frac{\rho+1}{5} .
$$

On the other hand,

$$
\begin{aligned}
& \left(\frac{1}{2 \rho^{2}}+\frac{\rho+1}{4}\right)^{2}-\left(1-\frac{1}{4 \rho^{2}}\right)\left(1-\frac{1}{\rho^{2}}\right) \\
& =\frac{\rho^{4}+2 \rho^{3}-15 \rho^{2}+4 \rho+24}{16 \rho^{2}} \\
& =\frac{(\rho+5)(\rho+1)(\rho-2)^{2}+4}{16 \rho^{2}}>0,
\end{aligned}
$$

which implies that

$$
\sqrt{\left(1-\frac{1}{4 \rho^{2}}\right)\left(1-\frac{1}{\rho^{2}}\right)}<\frac{1}{2 \rho^{2}}+\frac{\rho+1}{4} .
$$

Thus,

$$
h(\rho)<\frac{\rho+1}{4} .
$$

(3). It's easy to verify that when $\rho=\sqrt{\frac{3+\sqrt{13}}{2}}$,

$$
2 \sqrt{1-h(\rho)^{2}}=\sqrt{\rho^{2}-1} .
$$


Since $\sqrt{\rho^{2}-1}-2 \sqrt{1-h(\rho)^{2}}$ strictly increases on $[0,2]$, if $\rho<\sqrt{\frac{3+\sqrt{13}}{2}}$ then

$$
\sqrt{\rho^{2}-1}<2 \sqrt{1-h(\rho)^{2}}
$$

if $\sqrt{\frac{3+\sqrt{13}}{2}}<\rho \leq 2$ then

$$
\sqrt{\rho^{2}-1}>2 \sqrt{1-h(\rho)^{2}}
$$

Next, assume that $\rho>2$. Consider a right triangle $\triangle p u v$ with $\angle p u w=\frac{\pi}{2},\|p u\|=1$ and $\|p v\|=\rho$. Let $q$ be a point on the same side of $p v$ as $u$ such that $\|q v\|=\rho-1$ and $\|p q\|=\rho$. By Lemma 2, the distance between $q$ and $u v$ equals to $h(\rho)$. Since

$$
\|q u\|>\|p q\|-\|p u\|=\rho-1=\|q v\|,
$$

we have,

$$
\begin{aligned}
& \sqrt{\rho^{2}-1}=\|u v\|=\sqrt{\|q u\|^{2}-h(\rho)^{2}}+\sqrt{\|q v\|^{2}-h(\rho)^{2}} \\
& >2 \sqrt{(\rho-1)^{2}-h(\rho)^{2}}
\end{aligned}
$$

So, the third part of the lemma holds.

For each $\rho \geq 1$, let

$$
k(\rho)=\left\lceil\frac{\rho+1}{h(\rho)}\right\rceil .
$$

In the remaining of this section, we compute $k(\rho)$. It's easy to verify that $k(1)=4$. By Lemma $5(2)$, we have $k(\rho)=5$ on $(1,2]$. By Lemma $5(1)$, for any $\rho \geq 2$ we have

$$
\frac{\rho+1}{h(\rho)}>\frac{\rho+1}{\frac{\sqrt{3}}{2}(\rho-1)}>\frac{2}{\sqrt{3}} .
$$

By Lemma $4, \frac{\rho+1}{h(\rho)}$ is strictly decreasing on $[2, \infty)$. Hence

$$
\frac{2}{\sqrt{3}}<\frac{\rho+1}{h(\rho)} \leq \frac{2+1}{h(2)}<5 .
$$

Let $\rho_{1}, \rho_{2}$ and $\rho_{3}$ be the values greater than two at which $\frac{\rho+1}{h(\rho)}$ are equal to 4,3 and 2 respectively. Then,

$$
k(\rho)= \begin{cases}5 & \text { if } \rho \in\left(1, \rho_{1}\right) \\ 4 & \text { if } \rho=1 \text { or } \rho \in\left[\rho_{1}, \rho_{2}\right) ; \\ 3 & \text { if } \rho \in\left[\rho_{2}, \rho_{3}\right) \\ 2 & \text { if } \rho \in\left[\rho_{3}, \infty\right) .\end{cases}
$$

Next, we compute the numerical values of $\rho_{1}, \rho_{2}$ and $\rho_{3}$. A straightforward calculation yields that

$$
\begin{aligned}
\rho_{1}^{4}-2 \rho_{1}^{3}-\frac{21}{11} \rho_{1}^{2}+\frac{52}{11} \rho_{1}-\frac{24}{11} & =0 \\
\rho_{2}^{4}-\frac{56}{23} \rho_{2}^{3}-2 \rho_{2}^{2}+\frac{120}{23} \rho_{2}-\frac{57}{23} & =0 \\
\rho_{3}^{4}-4 \rho_{3}^{3}-\frac{5}{2} \rho_{3}^{2}+7 \rho_{3}-\frac{7}{2} & =0 .
\end{aligned}
$$

Using the quartic formula, we obtain

$$
\begin{aligned}
& \rho_{1}=\frac{1-\sqrt{\frac{25-c_{1}}{11}}+\sqrt{\frac{50+c_{1}}{11}+\frac{40}{\sqrt{11} \sqrt{25-c_{1}}}},}{2}, \\
& \rho_{2}=\frac{14-\sqrt{\frac{1117-c_{2}}{3}}+\sqrt{\frac{2234+c_{2}}{3}+\frac{2976}{\sqrt{\frac{1117-c_{2}}{3}}}},}{23} \\
& \rho_{3}=\sqrt{3 \sqrt{2}+\frac{33}{8}+\frac{\sqrt{2}}{4}+1,},
\end{aligned}
$$

where

$$
\begin{aligned}
& c_{1}=\sqrt[3]{2675+\frac{1280}{9} \sqrt{330}}+\sqrt[3]{2675-\frac{1280}{9} \sqrt{330}} \\
& c_{2}=23(\sqrt[3]{25021+2592 \sqrt{83}}+\sqrt[3]{25021-2592 \sqrt{83}}) .
\end{aligned}
$$

The numerical values of $\rho_{1}, \rho_{2}$ and $\rho_{3}$ are 2.0632, 2.5689, and 4.2462 respectively.

\section{STRIP COLORING}

In this section, we present an algorithm called strip coloring for computing a vertex coloring of the interference topology denoted by $H$. Our algorithm design exploits a key structural property of $H$. Recall that a graph $G=(V, E)$ is said to be a cocomparability graph if there is a vertex ordering $\left[v_{1}, v_{2}, \cdots, v_{n}\right]$ of $V$ such that if $i<j<k$ and $v_{i} v_{k} \in E$ then either $v_{i} v_{j} \in E$ or $v_{j} v_{k} \in E$. Such ordering is referred to as its cocomparability ordering. Every cocomparability graph is perfect [6], and its minimum vertex coloring can be computed in polynomial time by solving a maximum bipartite matching [5]. We discover that $H$ has the following restricted cocomparability:

Lemma 6: Let $U$ be any subset of of nodes which lie in a horizontal strip of height $h(\rho)$. Then, the subgraph $H[U]$ of $H$ induced by $U$ is a cocomparability graph with the lexicographic ordering being its cocomparability ordering.

Proof: Suppose that $v_{1}, v_{2}$ and $v_{3}$ are three nodes in $U$ in the lexicographic order satisfying that $v_{1}$ and $v_{3}$ interfere with each other. We prove by contradiction that either $v_{1}$ or $v_{3}$ interferes with $v_{2}$. Assume to the contrary that neither $v_{1}$ nor $v_{3}$ interferes with $v_{2}$. Then, both $\left\|v_{2} v_{1}\right\|$ and $\left\|v_{2} v_{3}\right\|$ are greater than $\max \{1, \rho-1\}$. Let $w$ be the intersection point between the segment $v_{1} v_{3}$ and the vertical line through $v_{2}$. Then,

$$
\left\|v_{2} w\right\| \leq h(\rho) \leq \max \{1, \rho-1\} .
$$

So $v_{2} w$ is shorter than both $v_{2} v_{1}$ and $v_{2} v_{3}$, which imply that both $\angle v_{2} v_{1} v_{3}$ and $\angle v_{2} v_{3} v_{1}$ are acute. Furthermore, the distance between $v_{2}$ and $v_{1} v_{3}$ is no more than $\left\|v_{2} w\right\|$, and hence is at most $h(\rho)$. By Lemma 5(1),

$$
\begin{aligned}
\left\|v_{1} v_{3}\right\| & >2 \sqrt{(\max \{1, \rho-1\})^{2}-h(\rho)^{2}} \\
& >\max \{1, \rho-1\} .
\end{aligned}
$$


Thus, either $D\left(v_{1}, 1\right) \cap D\left(v_{3}, \rho\right)$ contains at least one node other than $v_{1}$ or $D\left(v_{3}, 1\right) \cap D\left(v_{1}, \rho\right)$ contains at least one node other than $v_{3}$. By symmetry, we assume the former holds. We will prove that

$$
D\left(v_{1}, 1\right) \cap D\left(v_{3}, \rho\right) \subset D\left(v_{2}, \rho\right),
$$

which implies that $v_{2}$ and $v_{1}$ also interfere with each other.

Since $\left\|v_{1} v_{3}\right\|>\rho-1, \partial D\left(v_{1}, 1\right)$ and $\partial D\left(v_{3}, \rho\right)$ intersect at two points. Let $p$ be the intersection point of $\partial D\left(v_{1}, 1\right)$ and $\partial D\left(v_{3}, \rho\right)$ which lies on different side of $v_{1} v_{3}$ from $v_{2}$ (see Figure 5). Then,

$$
D\left(v_{1}, 1\right) \cap D\left(v_{3}, \rho\right) \subset D\left(v_{2}, \rho\right)
$$

if and only if $\left\|p v_{2}\right\| \leq \rho$. For $i=1$ and 3 , let $q_{i}$ be the point on $\partial D\left(v_{i}, \max \{1, \rho-1\}\right)$ satisfying that (1) the distance between $q_{i}$ and the line $v_{1} v_{3}$ is $h(\rho)$, (2) $q_{i}$ lies on the different side of $v_{1} v_{3}$ from $p$, and (3) the perpendicular foot $q_{i}^{\prime}$ of $q_{i}$ on the line $v_{1} v_{3}$ is on the segment $v_{1} v_{3}$. Since

$$
\begin{aligned}
\left\|v_{1} v_{3}\right\| & >2 \sqrt{(\max \{1, \rho-1\})^{2}-h(\rho)^{2}} \\
& =\left\|v_{1} q_{1}^{\prime}\right\|+\left\|v_{3} q_{3}^{\prime}\right\|,
\end{aligned}
$$

$q_{1}^{\prime}$ is closer to $v_{1}$ than $q_{3}^{\prime}$. Thus,

$$
\left\|v_{1} q_{3}\right\|>\left\|v_{1} q_{1}\right\|=\max \{1, \rho-1\} .
$$

This implies that $q_{3}$ is outside $D\left(v_{1}, \max \{1, \rho-1\}\right)$. Similarly, $q_{1}$ is outside $D\left(v_{3}, \max \{1, \rho-1\}\right)$. Then, $v_{2}$ lies inside the rectangle $q_{1} q_{1}^{\prime} q_{3}^{\prime} q_{3}$. So, $\left\|p v_{2}\right\| \leq \rho$ if the distances from $p$ to the four vertices of the rectangle $q_{1} q_{1}^{\prime} q_{3}^{\prime} q_{3}$ are all at most $\rho$. Clearly, for $i=1$ and 3 ,

$$
\left\|p q_{i}^{\prime}\right\| \leq \max \left\{\left\|p v_{1}\right\|,\left\|p v_{3}\right\|\right\}=\rho .
$$

Since both $p$ and $q_{1}$ lies on the same side of the perpendicular bisector of $v_{1} v_{3}$, which is also the perpendicular bisector of $q_{1} q_{3}$, we have $\left\|p q_{1}\right\| \leq\left\|p q_{3}\right\|$. Therefore, it is sufficient to show that $\left\|p q_{3}\right\| \leq \rho$. Consider the triangle $\triangle p v_{3} q_{3}$. Since $v_{3} q_{3}$ and $p v_{3}$ have fixed length, $\left\|p q_{3}\right\|$ strictly increases with $\angle p v_{3} q_{3}$ by the law of cosine. Since

$$
\angle p v_{3} q_{3}=\angle v_{1} v_{3} q_{3}+\angle v_{1} v_{3} p
$$

and $\angle v_{1} v_{3} q_{3}$ is also fixed, $\left\|p q_{3}\right\|$ also strictly increases with $\angle v_{1} v_{3} p$. We consider two cases.

Case 1: $1 \leq \rho<\sqrt{\frac{3+\sqrt{13}}{2}}$. By Lemma 5(3),

$$
2 \sqrt{(\max \{1, \rho-1\})^{2}-h(\rho)^{2}}>\sqrt{\rho^{2}-1} .
$$

Hence, $\angle p v_{1} v_{3}$ is always acute, and $\angle v_{1} v_{3} p$ strictly decreases with $\left\|v_{1} v_{3}\right\|$. So, $\left\|p q_{3}\right\|$ also strictly decreases with $\left\|v_{1} v_{3}\right\|$. By Lemma 1 , when $\left\|v_{1} v_{3}\right\|=2 \sqrt{1-h(\rho)^{2}},\left\|p q_{3}\right\|=\rho$. Thus, $\left\|p q_{3}\right\|<\rho$ in this case.

Case 2: $\rho \geq \sqrt{\frac{3+\sqrt{13}}{2}}$. By Lemma 5(3),

$$
2 \sqrt{(\max \{1, \rho-1\})^{2}-h(\rho)^{2}} \leq \sqrt{\rho^{2}-1}
$$

Thus, $\angle v_{1} v_{3} p$ achieves its maximum when $p v_{1}$ is perpendicular to $v_{1} v_{3}$. So, $\left\|p q_{3}\right\|$ achieves its maximum when $p v_{1}$ is

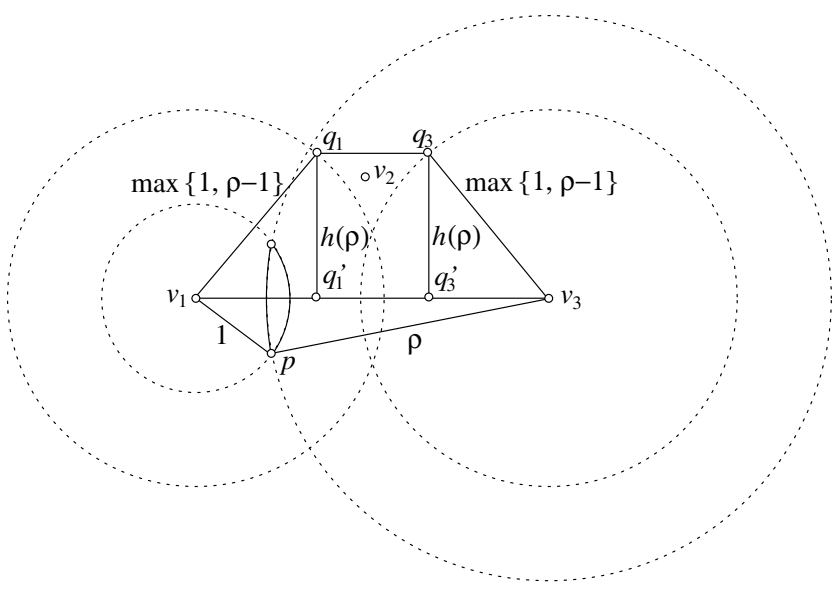

Fig. 5. Figure for the proof of Lemma 6 .

perpendicular to $v_{1} v_{3}$. By Lemma 2, the maximum of $\left\|p q_{3}\right\|$ is $\rho$. Thus, $\left\|p q_{3}\right\| \leq \rho$ in this case.

Now, we are ready to describe the algorithm strip coloring. For simplicity, each color is represented by a pair $\left(\lambda_{1}, \lambda_{2}\right)$ where $0 \leq \lambda_{1} \leq k(\rho)$ and $\lambda_{2}$ is a positive integer. We first compute the minimal axis-parallel rectangle surrounding all the networking nodes. Then, we partition such rectangle into top-closed bottom-open horizontal strips in the manner that the upper boundary of the top-most strip aligns with the top of the rectangle, the heights of all strips except the bottom-most one are all equal to $\frac{\rho+1}{k(\rho)}$, and the height of the bottom-most strip is at most $\frac{\rho+1}{k(\rho)}$ (see Figure 6). Number the successive strips from top to bottom using integers $0,1, \cdots$, and let $V_{i}$ denote the set nodes in the strip $i$. By the definition of $k(\rho)$, the height of each strip is at most $h(\rho)$. By Lemma 6, each $H\left[V_{i}\right]$ is a cocomparable graph and hence a minimum coloring of $H\left[V_{i}\right]$ with $\omega\left(H\left[V_{i}\right]\right)$ colors can be computed in polynomial time. So, we compute a minimum coloring of each $H\left[V_{i}\right]$ using the colors

$$
\left\{\left(i \bmod (k(\rho)+1), \lambda_{2}\right): 1 \leq \lambda_{2} \leq \omega\left(H\left[V_{i}\right]\right)\right\} .
$$

The entire coloring of all nodes is valid since for any $i$ and $j$ with $i<j$ and $(i-j) \bmod (k(\rho)+1)=0$, any node in a strip $i$ and any node in a strip $j$ are separated by a distance greater than $\rho+1$ and thus are not adjacent in $H$.

Next, we show that strip coloring uses at most $(k(\rho)+1) \omega(H)$ colors. Since $H\left[V_{i}\right]$ is a subgraph of $H$, $\omega\left(H\left[V_{i}\right]\right) \leq \omega(H)$. Therefore, all colors used by the strip coloring belong to the set

$$
\left\{\left(\lambda_{1}, \lambda_{2}\right): 0 \leq \lambda_{1} \leq k(\rho), 1 \leq \lambda_{2} \leq \omega(H)\right\} .
$$

This implies that the strip coloring uses at most $(k(\rho)+1) \omega(H)$ colors. In summary, we have the following theorem.

Theorem 7: The strip coloring uses at most $(k(\rho)+1) \omega(H)$ colors, and its approximation ratio is at most $k(\rho)+1$. 


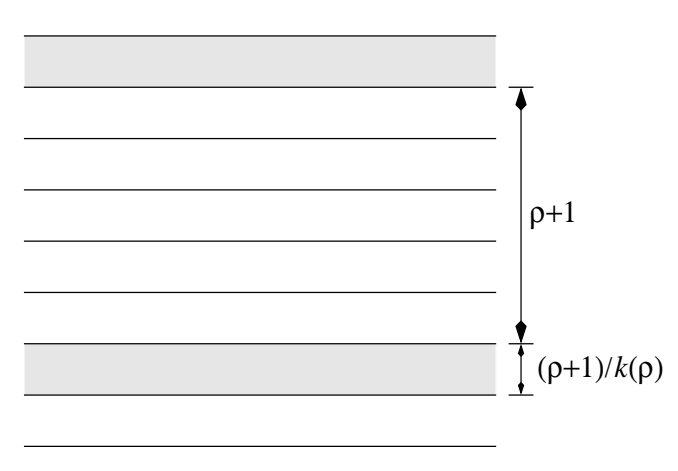

Fig. 6. Illustration of strip coloring.

Note that

$$
k(\rho)+1= \begin{cases}6 & \text { if } \rho \in\left(1, \rho_{1}\right) \\ 5 & \text { if } \rho=1 \text { or } \rho \in\left[\rho_{1}, \rho_{2}\right) \\ 4 & \text { if } \rho \in\left[\rho_{2}, \rho_{3}\right) \\ 3 & \text { if } \rho \in\left[\rho_{3}, \infty\right)\end{cases}
$$

For $\rho=1$, the strip coloring is a 5-approximation, an improvement upon the best-known 7 -approximation.

\section{Discussions}

We can still apply the greedy first-fit coloring on the interference topology $H$. For $\rho>1$, we can prove that its approximation ratio is bounded by a packing parameter $\eta(\rho)$ which is the maximal number of points whose pairwise distances are greater than $\max \{1, \rho-1\}$ in a half-disk of radius $\rho+1$. While still being bounded by a constant, $\eta(\rho)$ is at least 7 , which is worse than the bound $k(\rho)+1$ on the approximation ratio of the strip coloring.

Using the similar argument used in the proof of Lemma 6, we can prove that when all the networking nodes lie in a strip of height at most

$$
\max \{1, \rho-1\} \sqrt{1-\left(\frac{\max \{1, \rho-1\}}{2 \rho}\right)^{2}},
$$

the interference topology is cocomparable, and thus its minimum coloring can be solved in polynomial time.

\section{REFERENCES}

[1] I. Chlamtac and A. Farago, Making Transmission Schedules Immune to Topology Changes in Multi-Hop Packet Radio Networks, IEEE/ACM Transactions on Networking, Vol. 2, No. 1, pp. 23-29, Feb. 1994.

[2] I. Chlamtac and S. Kutten, A spatial reuse tdma/fdma for mobile multihop radio nertworks, in IEEE INFOCOM, pp. 389-394, March 1985.

[3] B.N. Clark, C.J. Colbourn, and D.S. Johnson. Unit disk graphs. Discrete Mathematics, 86:165-177, 1990.

[4] A. Ephremides and T.V. Truong, Scheduling Broadcasts in Multihop Radio Networks, IEEE Transactions on Communications, vol. 38, no. 4, pp. 456-60, April 1990.

[5] L. Ford and D. Fulkerson, Flows in Networks, Princeton University Press, Princeton, NJ, 1962.

[6] M.C. Golumbic. Algorithmic graph theory and perfect graphs. Academic Press, New York, NY, 1980.

[7] P. Gupta and P.Kumar, The capacity of wireless networks, IEEE Trans. Inform. Theory, vol. 46, pp. 388-404, Mar. 2000.

[8] D. W. Matula and L. L. Beck. Smallest-last ordering and clustering and graph coloring algorithms, Journal of the Association of Computing Machinery, 30(3):417-427, 1983.

[9] R.H. Möhring. Algorithmic aspects of comparability graphs and interval graphs. In I. Rival, editor, Graphs and Orders, pp. 41-101. Reidel, Dordrecht, 1985.

[10] R. Nelson and L. Kleinrock, Spatial-TDMA: A collision-free multihop channel access protocol, IEEE Transactions on Communications, vol. 33, no. 9, pp. 934944, Sep. 1985.

[11] S. Ramanathan and E.L. Lloyd, Scheduling algorithms for multi-hop radio networks, IEEE/ACM Transactions on Networking, vol. 1, pp. 166172, April 1993.

[12] R. Ramaswami and K. K. Parhi, Distributed scheduling of broadcasts in a radio network, in IEEE INFOCOM, pp. 497-504, 1989.

[13] A. Sen and M. L. Huson, A New Model for Scheduling Packet Radio Networks, ACM/Baltzer Journal Wireless Networks, 3 (1997), pp. 71-82.

[14] A. Sen and E. Malesinska, Approximation Algorithms for Radio Network Scheduling, Proceedings of 35th Allerton Coneference on Communication, Control and Computing, Champaign, Illinois, pp. 573-582, October, 1997.

[15] D.S. Stevens and M.H. Ammar, Evaluation of slot allocation strategies for TDMA protocols in packet radio networks, IEEE Military Communications Conference, pp: 835-839, 1990.

[16] P.-J. Wan, C.-W. Yi, X. Jia, and D. Kim: Approximation Algorithms for Conflict-Free Channel Assignment in Wireless Ad Hoc Networks, Wiley Journal on Wireless Communications and Mobile Computing, 6(2):201211, March 2006. 